\title{
Planar achromatic multiple beam splitter by adiabatic light transfer
}

\author{
Charles Ciret, ${ }^{1,2, *}$ Virginie Coda, ${ }^{1,2}$ Andon A. Rangelov, ${ }^{3}$ Dragomir N. Neshev, ${ }^{4}$ and Germano Montemezzani ${ }^{1,2}$ \\ ${ }^{1}$ Université de Lorraine, LMOPS, EA 4423, F-57070 Metz, France \\ ${ }^{2}$ Supélec, LMOPS, EA 4423, F-57070 Metz, France \\ ${ }^{3}$ Department of Physics, Sofia University, James Bourchier 5 Boulevard, 1164 Sofia, Bulgaria \\ ${ }^{4}$ Nonlinear Physics Centre and Centre for Ultrahigh-bandwidth Devices for Optical Systems (CUDOS), Research School of \\ Physics and Engineering, Australian National University, Canberra, ACT 0200, Australia \\ ${ }^{*}$ Corresponding author: charles.ciret@supelec.fr
}

Received July 9, 2012; accepted July 26, 2012;

posted August 3, 2012 (Doc. ID 171907); published September 6, 2012

\begin{abstract}
We introduce a novel achromatic and robust scheme for $n$-fold multiple beam splitting based on adiabatic light transfer in a planar geometry of coupled waveguides (WGs). The concept is experimentally verified for a one-to-three beam splitter by using a reconfigurable light-induced WG structure at two operating wavelengths. The demonstrated planar-type achromatic beam splitter opens new opportunities for the realization of ultra-high bandwidth on-chip photonic devices. (C) 2012 Optical Society of America

OCIS codes: $\quad 080.1238,230.1360,230.7390,130.3120,130.2790,190.5330$.
\end{abstract}

The formal analogy between wave coupling in adjacent optical waveguides (WGs) and the phenomenon of stimulated Raman adiabatic passage (STIRAP) in atomic physics [1,2] has inspired a number of recent studies. These are motivated both by the fundamental aspects of such optical phenomena and their potential application in integrated photonic devices [3-6] . All such devices, including beam splitters and couplers profit from the insensitivity of the adiabatic light transfer to the exact evolution of coupling coefficients between the WGs.

Beam splitters are among the most important devices in both bulk and integrated optics. They form the basic building blocks for many practical devices, and are critically essential for the realization of quantum logic gates in future quantum computers [7]. Conventional integrated beam splitters (for instance based on a directional coupler) are designed for a specific wavelength and generally suffer from chromatic limitations. STIRAP-like adiabatic light transfer can offer the solution to overcome this limitation. As such, one-to-three beam splitter based on multistate STIRAP [4] and a 1-to- $n$ multiple splitter based on a nonplanar three dimensional WG configuration [ $[6]$ have been theoretically proposed. Furthermore, a one-to-two achromatic beam splitter based on the optical analog to the fractional STIRAP process [8] has also been experimentally demonstrated using laser written WGs [9]. However, the development of on-chip high-bandwidth photonics requires an access to a larger number of output ports for the realization of robust and achromatic multiple beam splitters in planar geometry.

In this Letter, we propose theoretically and demonstrate experimentally a novel concept that exploits the evolution of an adiabatic transfer state [10] in the system and leads to multiple and achromatic splitting of light into $n$ output ports. In particular, we realize one-to-three planar beam splitter by using a reconfigurable lightinduced WG scheme [11] and test its performance for two different wavelengths of 633 and $850 \mathrm{~nm}$.

The WG structure leading to the multiple beam splitting is shown in Fig. 1(a). The structure is composed by a total of $N+2$ WGs. The input WG $I$ is placed near a buffer WG $B$ and an array of $N$ equidistant straight WGs. In the paraxial approximation in weakly curved WGs, the light evolution can be analyzed in the framework of the coupled mode theory with nearest neighbors coupling. By denoting $\mathbf{A}=\left(A_{I}, A_{B}, A_{1}, \ldots, A_{N}\right)^{T}$ the amplitude of the modes in the individual WGs, the propagation of a monochromatic wave in this structure can be described by

$$
i \frac{d}{d z} \mathbf{A}=\mathbf{H A}
$$

This is a set of $N+2$ differential equations where $\mathbf{H}$ is

$$
\mathbf{H}=\left(\begin{array}{cccccc}
0 & C_{P} & 0 & 0 & \ldots & 0 \\
C_{P} & 0 & C_{S} & 0 & \ldots & 0 \\
0 & C_{S} & 0 & C_{1,2} & \ldots & 0 \\
0 & 0 & C_{1,2} & 0 & \ddots & 0 \\
\vdots & \vdots & & \ddots & \ddots & C_{N-1, N} \\
0 & 0 & 0 & 0 & C_{N-1, N} & 0
\end{array}\right)
$$
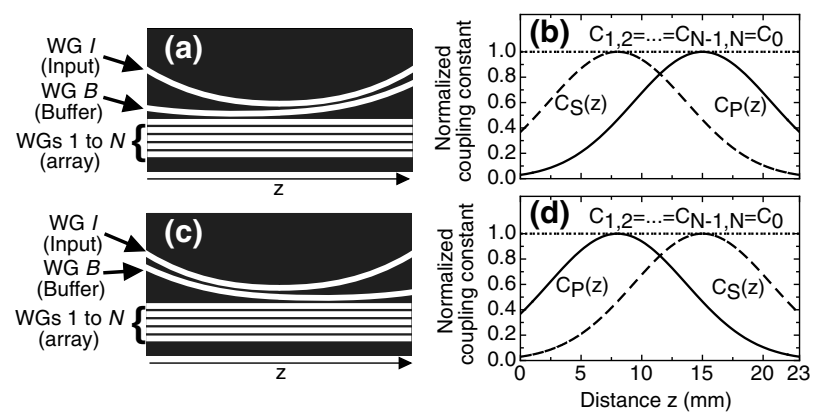

Fig. 1. (a) WG structure for multiple splitting of light (counterintuitive case) and (b) the corresponding longitudinal dependence of the normalized coupling constants. (c) and (d) The corresponding intuitive case, which does not lead to multiple beam splitting. 
The form of the WGs insures that the scalar coupling constants $C_{P}$ (between WG $I$ and WG $B$ ) and $C_{S}$ (between WG $B$ and WG 1 of the array) are $z$-dependent. In contrast, the coupling constants $C_{j, j+1} \quad(j=$ $1,2,3, \ldots, N-1$ ) between the WGs in the $N$-fold array are $z$ independent. As discussed later, if a multiple beam splitter with equal intensity outputs is desired all $C_{j, j+1}$ are chosen to be all equal to $C_{0}$. The quantities $C_{P}(z)$ and $C_{S}(z)$ are the analogues to the Rabi frequencies for the pump and Stokes pulses in the STIRAP process in quantum physics $[\underline{1}, 2,10]$. However, in contrast to STIR$\mathrm{AP}$ and its optical analogues [5,9], where the Stokes pulse couples the second-last and the last state in the chain, here $C_{S}(z)$ couples the second (WG $B$ ) and the third state (WG 1). The WG arrangement in Figs. 1(a) and 1(b) is said to be counterintuitive, as the coupling is strongest first among WGs that do not contain light. The intuitive order corresponds to the situation of Figs. 1(c) and 1(d). According to the STIRAP theory, for an odd number of WGs $N$ in the array the structure possesses an adiabatic transfer state, provided that the adiabatic condition $W \equiv Z \sqrt{C_{P m}^{2}+C_{S m}^{2}} \gg 1$ is fulfilled [2, 10]. Here $Z$ is the overlap distance of the coupling pulses $C_{P}(z)$ and $C_{S}(z)$, and $C_{P m}$ and $C_{S m}$ are their maximum values. The relative amplitude distribution at the output $(z=L)$ is [10]

$$
\frac{A_{2 k-1}}{A_{I}}=(-)^{k} \frac{C_{P}(L)}{C_{S}(L)} \prod_{j=1}^{k-1} \frac{C_{2 j-1,2 j}}{C_{2 j, 2 j+1}}=(-)^{k} \frac{C_{P}(L)}{C_{S}(L)},
$$

as well as $A_{2 k}(L)=0$ and $A_{B}(L)=0$. In (3) the second equality holds in our special case of a homogeneous WG array. Furthermore, in the counterintuitive case, for which $C_{P}(L)$ largely exceeds $C_{S}(L)$ [Fig. 1(b)], no light remains in WG $I$. Thus for an input field launched only in WG $I$, the output field will be

$$
\mathbf{A}(z=L)=\frac{1}{\sqrt{n}}(0,0,-1,0,1, \ldots,-1,0,1)^{T},
$$

where $n=(N+1) / 2$ is the number of excited output WGs (number of output ports). Figure 2(a) shows the convergence toward the amplitude distribution Eq. (4) for $N=5$ and a nearly ideal fulfillment of the adiabatic condition $(W=42)$, as calculated with Eq. (1). The corresponding expected evolutions under the conditions of the experiments discussed below are given in Figs. 2(b) and 2(c) for propagating wavelengths of 633 and $850 \mathrm{~nm}$. Importantly, the input light is divided into three essentially equally intense output ports, irrespective of the wavelength. It is worth noting that Eq. (3) also predicts that, if the coupling constants $C_{j, j+1}$ between the WGs of the array are not the same, different distributions of the intensities in the odd WGs of the array can be achieved. Figures $2(\mathrm{~d})$ and $2(\mathrm{e})$ show cases for which the multiple splitting does not work. Figure 2(d) is for an odd number of WGs $N$ but intuitive order of the coupling pulses as shown in Figs. 1(c) and 1(d). The light strongly oscillate between odd and even $\overline{\mathrm{WG}}$, which can be ascribed to Rabi-like oscillations [10]. Finally, for an even number
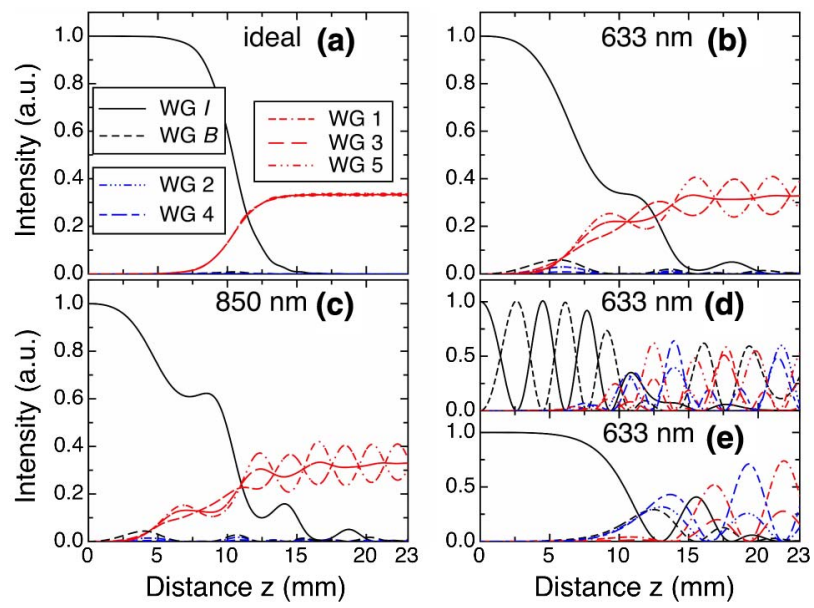

Fig. 2. (Color online) (a)-(c) Calculated light intensity evolution for $N=5$ and counterintuitive case. (a) Nearly ideal adiabatic condition ( $W=42)$; (b) and (c) simulate our experimental case for light at $633 \mathrm{~nm}(W \approx 12.4)$ and $850 \mathrm{~nm}(W \approx 17)$, respectively; (d) is for $N=5$ but intuitive order of $C_{P}(z)$ and $C_{S}(z)$. (e) Even number of WGs $(N=4)$ and counterintuitive order.

of WGs the STIRAP theory predicts that an adiabatic transfer state does not exist [10], resulting in a complex light evolution as seen in Fig. 2(e).

Experimental verification of the above predicted multiple splitting is performed by photoinducing the WG structures of Figs. 1(a) and 1(c) using lateral illumination of a biased photorefractive crystal $[11,12]$. A simplified setup is shown in Fig. 3. A 23 mm-long weakly Ce doped $\mathrm{Sr}_{0.61} \mathrm{Ba}_{0.39} \mathrm{Nb}_{2} \mathrm{O}_{6}$ crystal (SBN) is used to record the WGs. The desired optical structure is imprinted onto a $532 \mathrm{~nm} \mathrm{cw}$ control beam by a spatial light modulator (SLM) and imaged on the crystal by two crossed cylindrical lenses. The photorefractive process in the crystal takes place under a bias electric field $E_{0}$ and leads to local refractive index changes $\Delta n$, which depend on $E_{0}$ and reproduce the structure on the control beam. Easy reconfiguration of the WG structure is achieved by changing the pattern on the SLM. The structure is probed by a low power beam at two different wavelengths (633 and $850 \mathrm{~nm}$ ) allowing to verify the achromaticity of our adiabatic beam splitter scheme. The probe beam output intensity distribution is observed by imaging the exit face of the crystal on a CCD camera. A Mach-Zehnder interferometer is used to reveal the relative phase between

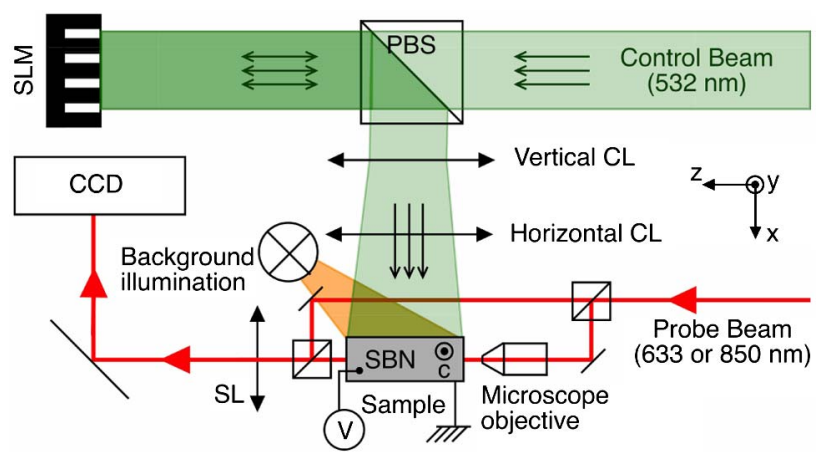

Fig. 3. (Color online) Setup for photoinducing the desired structures. SLM: spatial light modulator, CL: cylindrical lens, SL: spherical lens, PBS: polarizing beam splitter. 

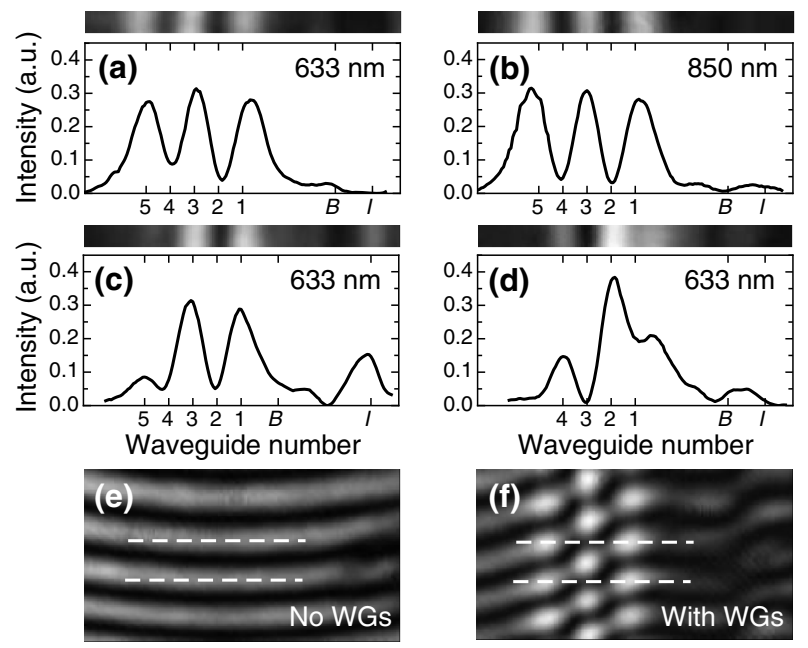

Fig. 4. (a) and (b) Output intensity profiles for counterintuitive order, $N=5$ at $\lambda=633$ and $850 \mathrm{~nm}$, respectively, showing achromatic beam splitting. (c) Intuitive order, $N=5$ and $\lambda=633 \mathrm{~nm}$. (d) Counterintuitive order, $N=4$. (e) Probe beam interferogram in the absence of WGs and (f) with induced WGs showing $\pi$ phase shift between the output ports.

the output ports. In our experiments the WG width is $7.2 \mu \mathrm{m}$ with an index modulation $\Delta n \approx 10^{-4}$ as a result of the application of a bias voltage of $E_{0}=3 \mathrm{kV} / \mathrm{cm}$. The array has a period of $9.6 \mu \mathrm{m}$. The effective value [13] of the maximum coupling constant $C_{0}$ is $C_{0}$ $(633 \mathrm{~nm})=1.1 \pm 0.1 \mathrm{~mm}^{-1}$ and $C_{0}(850 \mathrm{~nm})=1.5 \pm$ $0.1 \mathrm{~mm}^{-1}$, leading to $W \approx 12.4$ at $633 \mathrm{~nm}$ and $W \approx 17$ at $850 \mathrm{~nm}$. The coupling constants have been estimated by analyzing the extent of the discrete diffraction patterns [14] in a WG array composed of 150 WGs with the same WG parameters.

Our experimental results are summarized in Fig. 4. As seen in Figs. 4(a) and 4(b), for an odd number of WĞs in the array $(N=5)$ and the counterintuitive order, light splits into three nearly equal output ports, where the odd WGs are populated, while the even number of WGs have negligible intensity. Most importantly, the adiabatic process is completely independent on the excitation wavelength, thus confirming the theoretical expectations and proving the achromaticity of the process. As expected, in all other cases including the intuitive order (Fig. 4(c)) or structure with even number of WGs (Fig. 4(d) ) the wished light splitting does not occur and the output pattern is more complex. The solution (4) for the output amplitudes of the adiabatic transfer state under our conditions predicts that the waves in neighboring activated ports of the array are mutually out of phase by $\pi$. Figs. $\underline{4(\mathrm{e})}$ and $\underline{4(\mathrm{f})}$ compare the interferograms in absence of induced WGs and for the geometry used in Fig. 4(a), with three output ports (vertical stripes in the planar-type WGs). The observed $\pi$ phase shift in Fig. $\underline{4(\mathrm{f})}$ between the central (WG 3) and the two other stripes (WG 1 and WG 5) fully confirms the theoretical expectations.

In conclusion, we have presented and verified experimentally a new concept for multiple and highly achromatic light splitting based on adiabatic light transfer. Unlike for earlier proposals based on adiabatic light transfer $[\underline{4}, \underline{6}, \underline{9}]$, here equal intensity splitting does not rely on specific design for intermediate coupling constants, on specific three-dimensional WG arrangements, or on a specific propagation distance. Provided that the adiabatic condition is well fulfilled, the present planartype approach allows robust and achromatic wave splitting into a large number of output ports, suitable for applications in ultra-high bandwidth on-chip photonics. Moreover, our approach can be generalized to provide arbitrary splitting distributions at the device output by changing the coupling constants between the WGs of the array through the WG spacing.

DNN thanks A. Greentree for the useful discussions.

\section{References}

1. U. Gaubatz, P. Rudecki, S. Schiemann, and K. Bergmann, J. Chem. Phys. 92, 5363 (1990).

2. N. V. Vitanov, M. Fleischer, B. W. Shore, and K. Bergmann, Adv. At. Mol. Opt. Phys. 46, 55 (2001).

3. S. Longhi, Laser Photon. Rev. 3, 243 (2009).

4. S. Y. Tseng and M. C. Wu, J. Lightwave Technol. 28, 3529 (2010).

5. G. Della Valle, M. Ornigotti, T. Toney Fernandez, P. Laporta, S. Longhi, A. Coppa, and V. Foglietti, Appl. Phys. Lett. 92, 011106 (2008).

6. A. A. Rangelov and N. V. Vitanov, Phys. Rev. A 85, 055803 (2012).

7. J. L. O'Brien, G. J. Pryde, A. G. White, T. C. Ralph, and D. Branning, Nature 426, 264 (2003).

8. N. V. Vitanov, K. A. Suominen, and B. W. Shore, J. Phys. B 32, 4535 (1999).

9. F. Dreisow, M. Ornigotti, A. Szameit, M. Heinrich, R. Keil, S. Nolte, A. Tünnermann, and S. Longhi, Appl. Phys. Lett. 95, 261102 (2009).

10. N. V. Vitanov, Phys. Rev. A 58, 2295 (1998).

11. Ph. Dittrich, G. Montemezzani, P. Bernasconi, and P. Günter, Opt. Lett. 24, 1508 (1999).

12. M. Gorram, V. Coda, P. Thévenin, and G. Montemezzani, Appl. Phys. B 95, 565 (2009).

13. N. Belabas, C. Minot, J. A. Levenson, and J. M. Moison, J. Lightwave Technol. 29, 3009 (2011).

14. F. Lederer, G. I. Stegeman, D. N. Christodoulides, G. Assanto, M. Segev, and Y. Silberberg, Phys. Rep. 463, 1 (2008). 